OPEN ACCESS

Edited by:

Ziad Daoud,

Central Michigan University,

United States

Reviewed by:

Deepjyoti Paul,

Assam University, India

Aldert Zomer,

Utrecht University, Netherlands

*Correspondence:

Dilruba Ahmed

dahmed@icddrb.org

Dinesh Mondal

din63d@icddrb.org

tThese authors have contributed equally to this work and share first authorship

Specialty section:

This article was submitted to Antimicrobials, Resistance

and Chemotherapy,

a section of the journal

Frontiers in Microbiology

Received: 05 July 2021 Accepted: 03 September 2021

Published: 04 October 2021

Citation:

Mazumder R, Hussain A, Abdullah A, Islam MN, Sadique MT, Muniruzzaman SM, Tabassum A,

Halim F, Akter N, Ahmed D and Mondal D (2021) International

High-Risk Clones Among Extended-Spectrum

$\beta$-Lactamase-Producing Escherichia

coli in Dhaka, Bangladesh.

Front. Microbiol. 12:736464.

doi: 10.3389/fmicb.2021.736464

\section{International High-Risk Clones Among Extended-Spectrum $\beta$-Lactamase-Producing Escherichia coli in Dhaka, Bangladesh}

Razib Mazumder't, Arif Hussain ${ }^{1+}$, Ahmed Abdullah', Md. Nazrul Islam², Md. Tuhin Sadique ${ }^{2}$, S. M. Muniruzzaman², Anika Tabassum², Farhana Halim², Nasrin Akter $^{2}$, Dilruba Ahmed ${ }^{2 *}$ and Dinesh Mondal ${ }^{*}$

\begin{abstract}
1 Genomics Center, Laboratory Sciences and Services Division, International Centre for Diarrhoeal Disease Research, Bangladesh (icddr,b), Dhaka, Bangladesh, ${ }^{2}$ Clinical Microbiology and Immunology Laboratory, Laboratory Sciences and Services Division, International Centre for Diarrhoeal Disease Research, Bangladesh (icddr,b), Dhaka, Bangladesh
\end{abstract}

Background: Escherichia coli is a major extended-spectrum $\beta$-lactamase (ESBL)producing organism responsible for the rapid spread of antimicrobial resistance (AMR) that has compromised our ability to treat infections. Baseline data on population structure, virulence, and resistance mechanisms in E. coli lineages from developing countries such as Bangladesh are lacking.

Methods: Whole-genome sequencing was performed for $46 \mathrm{ESBL}-E$. coli isolates cultured from patient samples at the International Centre for Diarrhoeal Disease Research, Bangladesh (icddr,b)-Dhaka. Sequence data were analyzed to glean details of AMR, virulence, and phylogenetic and molecular markers of $E$. coli lineages.

Results: Genome comparison revealed presence of all major high-risk clones including sequence type 131 (ST131) (46\%), ST405 (13\%), ST648 (7\%), ST410 (4.3\%), ST38 (2\%), ST73 (2\%), and ST1193 (2\%). The predominant ESBL gene and plasmid combination were bla $\mathrm{CTX}-\mathrm{M}-15$ and FII-FIA-FIB detected in diverse E. coli phylogroups and STs. The blanDM-5 (9\%) gene was present in prominent E. coli STs. One (2\%) mcr-1-positive ST1011 E. coli, coharboring bla ${ }_{\mathrm{CTXM}-55}$ gene, was detected. The extraintestinal pathogenic E. coli genotype was associated with specific E. coli lineages. The single nucleotide polymorphism (SNP)-based genome phylogeny largely showed correlation with phylogroups, serogroups, and fimH types. Majority of these isolates were susceptible to amikacin (93\%), imipenem (93\%), and nitrofurantoin (83\%).

Conclusion: Our study reveals a high diversity of E. coli lineages among ESBLproducing $E$. coli from Dhaka. This study suggests ongoing circulation of ST131 and all major non-ST131 high-risk clones that are strongly associated with cephalosporin resistance and virulence genes. These findings warrant prospective monitoring of high-risk clones, which would otherwise worsen the AMR crises.

Keywords: CTX-M, NDM, MCR, extraintestinal pathogenic E. coli (ExPEC), carbapenem resistance, wholegenome sequencing, epidemiological successful clones, ST131 and non-ST131 lineages 


\section{INTRODUCTION}

Infections with Escherichia coli that produce extended-spectrum $\beta$-lactamases (ESBLs) present an increasing clinical and public health threat (Mathers et al., 2015). These bacteria are resistant to several new-generation cephalosporin agents and render them ineffective for treating infections (Pitout and Laupland, 2008). Infections of ESBL E. coli causes increased morbidity, high mortality, longer hospital stays, and increased health care costs in comparison to infections caused by non-ESBL E. coli (Paterson and Bonomo, 2005). Treatment of such infections has been further complicated by the emergence of carbapenem resistance in ESBL-E. coli; these carbapenem-resistant isolates are often found resistant to all the available antibiotics (Doi, 2019).

Several strains of ESBL-producing E. coli are pathogenic; they commonly cause urinary tract infections (UTIs) in otherwise healthy people (Calbo et al., 2006; Franz et al., 2015). Moreover, when certain pathogenic bacterial clones horizontally acquire ESBL genes, they can emerge and reemerge rapidly within the population through clonal dissemination and thereby gain local or even global predominance as international high-risk clones (Price et al., 2013). Examples of such epidemiological successful extraintestinal pathogenic E. coli (ExPEC) lineages include sequence type 131 (ST131), ST410, ST38, ST73, ST405, and ST648, which are associated with both nosocomial and community-acquired infections and are being increasingly detected from multiple origins, worldwide (Baquero et al., 2013; Shaik et al., 2017; Manges et al., 2019). Since the last decade, CTXM-type enzymes have become the most predominant ESBLs in E. coli and other Gram-negative bacteria of clinical significance (Cantón and Coque, 2006). Similarly, the transposable elements containing the New Delhi metallo- $\beta$-lactamase (NDM) gene have been identified to be responsible for the rapid dissemination of carbapenem resistance in E. coli (Kumarasamy et al., 2010).

Currently, a high number of ESBL-producing E. coli infections are linked to the pandemic E. coli lineage ST131 (NicolasChanoine et al., 2008). Moreover, it is shown that E. coli ST131 strains are strongly associated with CTX-M-15-type ESBL and are predominantly responsible for causing bladder infections, kidney infections, and urosepsis worldwide including Southeast Asia (Nicolas-Chanoine et al., 2014; Chen et al., 2019). For instance, in India, it was reported that $93 \%$ of ST131 E. coli isolates carried CTX-M-15. Studies have also reported the presence of the $H 30$ subclone that is reportedly responsible for the clonal dissemination of ST131 E. coli (Hussain et al., 2012, 2014; Ranjan et al., 2015; Shaik et al., 2017). A study from Bangladesh reported that $71 \%$ of ESBL-producing E. coli was linked to the ST131 clone (Begum and Shamsuzzaman, 2016). Another study from Bangladesh identified a clonal cluster of clinical E. coli isolates belonging to serotype $\mathrm{O} 25: \mathrm{H} 4$, which indicates the widespread circulation of the ST131 E. coli lineage (Lina et al., 2014).

High-resolution studies on ESBL-producing E. coli strains from Bangladesh are lacking compared with the rest of the world, which limit our understanding of the population structure, emerging antimicrobial resistance (AMR) and their potential to spread. Herein, we report the findings of a genomic epidemiological investigation of the recent ESBL-E. coli isolates, especially the CTX-M-type ESBL producers associated with extraintestinal infections in Dhaka, Bangladesh. We analyzed their resistance genes, virulence genes, plasmid types, phylogenetic relatedness, and molecular features. Our study suggests ongoing circulation of ST131 and all major non-ST131 E. coli high-risk clones in Dhaka, Bangladesh. Studies such as these will provide important insights into the evolution of pathogens and will inform novel interventional strategies.

\section{MATERIALS AND METHODS}

\section{Study Setting, Bacterial Isolates, and Antimicrobial Susceptibility}

Bacterial isolates were collected randomly as part of routine (1\%) surveillance (128 E. coli isolates) between March 2018 and July 2019 from the Clinical Microbiology and Immunology Laboratory of the International Center for Diarrheal Disease Research, Bangladesh (icddr,b) (Mazumder et al., 2020a). From this collection of 128 E. coli isolates, 46 randomly selected ESBL E. coli spanning over the entire 16-month period (representing 1-4 isolates per month) were whole genome sequenced. Of these 46 isolates, 34 were obtained from urine and 12 from pus specimen (Table 1). Identification of bacterial isolates was performed using standard biochemical methods. Antimicrobial susceptibility testing by disk diffusion was performed against the following antibiotics: gentamicin $(10 \mu \mathrm{g})$, amikacin $(30 \mu \mathrm{g})$, cotrimoxazole $(25 \mu \mathrm{g})$, ciprofloxacin $(5 \mu \mathrm{g})$, nitrofurantoin $(200 \mu \mathrm{g})$, cefuroxime $(30 \mu \mathrm{g})$, ceftriaxone $(30 \mu \mathrm{g})$, cefixime $(5 \mu \mathrm{g})$, cefepime $(30 \mu \mathrm{g})$, and imipenem $(10 \mu \mathrm{g})$. E. coli ATCC 25922 was used for quality control (Clinical and Laboratory Standards Institute, 2015).

\section{Whole-Genome Sequencing, Genome Assembly, and Annotation}

Genomic DNA from an overnight bacterial culture was extracted and purified using QIAamp DNA Mini kit (Qiagen, Germany). The purity of the genomic DNA was assessed using a NanoDrop spectrophotometer (Thermo Fisher Scientific, United States), and quantification was performed using a Qubit 2.0 fluorometer (Life Technologies). DNA libraries were prepared using the Nextera XT DNA library Prep kit (Illumina) (Mazumder et al., 2020b). The pooled library was sequenced at the genomic sequencing facility of icddr,b on an Illumina Nextseq500 system using the 150-base-paired-end Mid-output v2.5 sequencing kit. The sequence reads were trimmed and filtered using Trimmomatic 0.39 (Bolger et al., 2014), and de novo assemblies of the resulting reads were generated using SPAdes 3.11.1 (Bankevich et al., 2012), and the quality check was done using QUAST v5.02 (Mikheenko et al., 2018). The genomes of our study were annotated using Prokka de novo (Seemann, 2014). The program was run on fast mode, and the genus-specific BLAST database was used. The genome features and associated metadata are listed in Table $\mathbf{1 .}$

\section{In silico Molecular Analysis}

Phylotyping was done using the ClermonTyping online tool (Beghain et al., 2018). Other molecular features were 
TABLE 1 | Genome features and metadata of 46 ESBL E. coli isolates sequenced in this study.

\begin{tabular}{|c|c|c|c|c|c|c|c|c|c|}
\hline SI no. & Isolate ID & Date of isolation & Source & STs & Genome coverage & Accession no. & Contig no. (>200 bp) & Genome size (bp) & $\begin{array}{c}\text { No. of Coding } \\
\text { Sequences (CDS) }\end{array}$ \\
\hline 1 & LMLEEc001 & 20-Apr-2018 & Urine & 131 & $83.9 x$ & JACHQR000000000.1 & 104 & $5,220,525$ & 4,897 \\
\hline 2 & LMLEEc002 & 3-May-2018 & Urine & 3,748 & $91.4 X$ & JACHQQ000000000.1 & 160 & $5,079,033$ & 4,793 \\
\hline 3 & LMLEEc003 & 3-May-2018 & Pus & 131 & $91.4 X$ & JACHQP000000000.1 & 199 & $5,210,796$ & 4,887 \\
\hline 4 & LMLEEc010 & 2-Jun-2018 & Urine & 131 & $95.4 X$ & JACHQO000000000.1 & 112 & $5,299,824$ & 5,029 \\
\hline 5 & LMLEEc018 & 28-Jun-2018 & Pus & 410 & $92.3 x$ & JACHQN000000000.1 & 98 & $4,888,814$ & 4,570 \\
\hline 6 & LMLEEc019 & 28-Jun-2018 & Pus & 410 & $97.8 X$ & JACHQM000000000.1 & 99 & $4,888,382$ & 4,566 \\
\hline 7 & LMLEEc020 & 5-Jul-2018 & Urine & 167 & $88.6 X$ & JACHQL000000000.1 & 150 & $4,963,734$ & 4,655 \\
\hline 8 & LMLEEc025 & 19-Jul-2018 & Urine & 131 & $113.5 X$ & JACHQK000000000.1 & 119 & $5,076,042$ & 4,785 \\
\hline 9 & LMLEEc027 & 26-Jul-2018 & Urine & 131 & $62.9 x$ & JACHQJ000000000.1 & 123 & $5,211,152$ & 4,905 \\
\hline 10 & LMLEEc029 & 2-Aug-2018 & Urine & 131 & $67 X$ & JACHQI000000000.1 & 97 & $5,234,461$ & 4,966 \\
\hline 11 & LMLEEc033 & 16-Aug-2018 & Urine & 162 & $71.4 X$ & JACHQH000000000.1 & 113 & $5,004,684$ & 4,677 \\
\hline 12 & LMLEEc034 & 24-Aug-2018 & Urine & 131 & $71.4 X$ & JACHQG000000000.1 & 113 & $5,261,112$ & 4,939 \\
\hline 13 & LMLEEc035 & 23-Aug-2018 & Pus & 648 & $65.4 X$ & JACHQF000000000.1 & 188 & $5,376,792$ & 5,053 \\
\hline 14 & LMLEEc036 & 31-Aug-2018 & Urine & 131 & $73.2 X$ & JACHQE000000000.1 & 86 & $5,237,487$ & 4,928 \\
\hline 15 & LMLEEc039 & 6-Sep-2018 & Urine & 648 & $83.2 X$ & JACHQD000000000.1 & 118 & $5,119,774$ & 4,741 \\
\hline 16 & LMLEEc040 & 13-Sep-2018 & Urine & 131 & $65.6 \mathrm{X}$ & JACHQC000000000.1 & 122 & $5,263,046$ & 4,943 \\
\hline 17 & LMLEEc041 & 13-Sep-2018 & Urine & 1,193 & $73.7 X$ & JACHQB000000000.1 & 82 & $5,023,339$ & 4,696 \\
\hline 18 & LMLEEc043 & 20-Sep-2018 & Urine & 131 & $62.1 X$ & JACHQA000000000.1 & 92 & $4,972,440$ & 4,633 \\
\hline 19 & LMLEEc050 & 18-Oct-2018 & Urine & 131 & $54.6 x$ & JACHPZ000000000.1 & 92 & $5,237,567$ & 4,926 \\
\hline 20 & LMLEEc056 & 8-Nov-2018 & Urine & 405 & $62.2 X$ & JACHPY000000000.1 & 149 & $5,278,053$ & 4,880 \\
\hline 21 & LMLEEc059 & 15-Nov-2018 & Urine & 405 & $66.8 X$ & JACHPX000000000.1 & 156 & $5,348,292$ & 4,939 \\
\hline 22 & LMLEEc062 & 29-Nov-2018 & Urine & 405 & $67.4 X$ & JACHPW000000000.1 & 129 & $5,228,791$ & 4,886 \\
\hline 23 & LMLEEc063 & 29-Nov-2018 & Urine & 131 & $62.5 X$ & JACHPV000000000.1 & 83 & $5,180,621$ & 4,908 \\
\hline 24 & LMLEEc064 & 6-Dec-2018 & Urine & 2,851 & $53 X$ & JACHPU000000000.1 & 156 & $5,180,445$ & 4,969 \\
\hline 25 & LMLEEc070 & 29-Dec-2018 & Pus & 2,178 & $83.2 X$ & JACHPT000000000.1 & 90 & $4,980,462$ & 4,708 \\
\hline 26 & LMLEEc071 & 29-Dec-2018 & Pus & 131 & $73.1 \mathrm{x}$ & JACHPS000000000.1 & 65 & $5,110,507$ & 4,817 \\
\hline 27 & LMLEEc072 & 3-Jan-2019 & Urine & 131 & $76.9 x$ & JACHPR000000000.1 & 113 & $5,248,087$ & 4,933 \\
\hline 28 & LMLEEc074 & 10-Jan-2019 & Urine & 131 & $89.8 X$ & JACHPQ000000000.1 & 121 & $5,315,415$ & 5,069 \\
\hline 29 & LMLEEc078 & 24-Jan-2019 & Urine & 421 & $143.1 X$ & JACHPP000000000.1 & 106 & $5,087,660$ & 4,788 \\
\hline 30 & LMLEEc082 & 14-Feb-2019 & Urine & 131 & $89.8 X$ & JACHPO000000000.1 & 109 & $5,282,720$ & 5,015 \\
\hline 31 & LMLEEc087 & 21-Feb-2019 & Urine & 131 & $67.9 x$ & JACHPN000000000.1 & 115 & $5,197,822$ & 4,882 \\
\hline 32 & LMLEEc088 & 28-Feb-2019 & Urine & 648 & $73.9 x$ & JACHPM000000000.1 & 135 & $5,193,220$ & 4,906 \\
\hline 33 & LMLEEc089 & 28-Feb-2019 & Urine & 405 & $62.7 X$ & JACHPL000000000.1 & 166 & $5,359,628$ & 5,003 \\
\hline 34 & LMLEEc091 & 6-Mar-2019 & Urine & 617 & $50.6 x$ & JACHPK000000000.1 & 143 & $5,042,428$ & 4,717 \\
\hline 35 & LMLEEc097 & 28-Mar-2019 & Urine & 131 & $68.2 X$ & JACHPJ000000000.1 & 125 & $5,354,581$ & 5,109 \\
\hline 36 & LMLEEc101 & 11-Apr-2019 & Pus & 48 & $77.9 x$ & JACHPI000000000.1 & 121 & $4,535,056$ & 4,220 \\
\hline 37 & LMLEEc103 & 18-Apr-2019 & Pus & 131 & $85 X$ & JACHPH000000000.1 & 106 & $5,233,284$ & 4,952 \\
\hline 38 & LMLEEc104 & 25-Apr-2019 & Urine & 131 & $69.4 X$ & JACHPG000000000.1 & 100 & $5,277,281$ & 5,004 \\
\hline 39 & LMLEEc108 & 9-May-2019 & Urine & 405 & $62.4 X$ & JACHPF000000000.1 & 173 & $5,515,065$ & 5,131 \\
\hline 40 & LMLEEc111 & 23-May-2019 & Urine & 38 & $63.4 X$ & JACHPE000000000.1 & 93 & $4,982,213$ & 4,587 \\
\hline 41 & LMLEEc115 & 13-Jun-2019 & Pus & 73 & $78.7 X$ & JACHPD000000000.1 & 138 & $5,149,754$ & 4,752 \\
\hline 42 & LMLEEc117 & 7-Jun-2019 & Urine & 354 & $56.4 X$ & JACHPC000000000.1 & 93 & $5,346,716$ & 5,078 \\
\hline 43 & LMLEEc120 & 27-Jun-2019 & Urine & 131 & $49.8 X$ & JACHPB000000000.1 & 159 & $5,245,925$ & 4,948 \\
\hline 44 & LMLEEc123 & 6-Jul-2019 & Pus & 1,011 & $79 x$ & JACHPA000000000.1 & 140 & $5,218,770$ & 4,823 \\
\hline 45 & LMLEEc125 & 11-Jul-2019 & Pus & 405 & $76.5 x$ & JACHOZOO00000000.1 & 216 & $5,514,459$ & 5,180 \\
\hline 46 & LMLEEc127 & 20-Jul-2019 & Pus & 1,884 & $68.2 X$ & JACHOY000000000.1 & 72 & $5,050,573$ & 4,666 \\
\hline
\end{tabular}

identified using the Center for Genomic Epidemiology's Bioinformatics tools using default parameters unless otherwise stated. Specifically, MLST 2.0 (Larsen et al., 2012) was used to identify STs, SerotypeFinder 1.1 (Joensen et al., 2015) for detecting serotypes, FimTyper 1.0 (Roer et al., 2017) to determine fimH types, and PlasmidFinder 2.1 (Carattoli et al., 2014) for identification of plasmid incompatibility groups. Comprehensive Antibiotic Resistance Database (McArthur et al., 2013) was used for screening the presence of acquired AMR genes; virulence genes were detected using a custom-made database of 50 genes from the Virulence Factors Database (Chen et al., 2005) belonging to different categories as described previously 
(Stoesser et al., 2015), a threshold, $\geq 90 \%$ identity; minimum coverage, $\geq 70 \%$ was used for both. AMR-related chromosomal point mutations were identified in the draft genomes using PointFinder (Zankari et al., 2017). The genetic context of $b l a_{\mathrm{CTX}-\mathrm{M}-15}, b l a_{\mathrm{NDM}-5}$, and $m c r-1$ with respect to plasmid was determined by BLAST searching of particular contigs against the GenBank database. Genomes having three or more ExPEC-associated genes were classified as ExPEC according to Johnson's criteria (Johnson and Stell, 2000). From virulence and resistance gene presence and absence CSV files, heat plots were obtained, using the Python module seaborn and matplotlib.

\section{Single Nucleotide Polymorphism-Based Core Genome Phylogeny}

The reference (E. coli 0154:H7 Sakai strain) guided multifasta consensus alignment of $46 \mathrm{E}$. coli genomes was obtained using the Snippy v4.4.0 pipeline (Seemann, 2015). Putative recombination regions were detected and masked using Gubbins v2.3.4 (Croucher et al., 2015). Finally, the SNP- based phylogeny was inferred using RaxML v8.2.12 (Stamatakis, 2014) using GTR (Generalized Time Reversible) substitution model and GAMMA distribution as the model of rate heterogeneity.

\section{Statistical Analysis}

Using SPSS statistics software (version 25.0), continuous variables were compared with the non-parametric Mann-Whitney $U$ test, and proportions were analyzed using the $\chi^{2}$-test; $p$-values with a threshold of $<0.05$ were considered statistically significant. Accordingly, the $p$-values are indicated within text where appropriate.

\section{RESULTS}

\section{Molecular Typing of Extended-Spectrum $\beta$-Lactamase Escherichia coli Isolates}

The sequence diversity of the study isolates as analyzed by in silico MLST demonstrates that the 46 ESBL-E. coli isolates belonged to 19 different STs (Figure 1). The pandemic ST131 lineage was the most predominant ST identified, comprising 46\% (21/46) of the isolates. Apart from ST131, the other significant STs included the presence of isolates belonging to the international high-risk clones, such as ST405 (13\% 6/46), ST648 (7\% 3/46), ST410 (4.3\% 2/46) ST38 (2\%), ST73 (2\%), and ST1193 (2\%). Phylogrouping identified a total of six phylogroups, namely, A, B1, B2, C, D, and F, in the ESBL-E. coli isolates (Figure 1). The phylogroup B2 (24/46) was the major phylogroup in our collection, which predominantly consisted of ST131 isolates (88\%, 21/24). Phylogroup D was the second largest phylogroup with $20 \%$ isolates ( $n=9,6$ ST405, 1 ST38, 1 ST1011, 1 ST1884), which was followed by group $\mathrm{F}(n=4,3$ ST648, 1 ST354), B1 $(n=3,1$ ST3748, 1 ST162, 1 ST2178), C $(n=3,2$ ST410, 1 ST2851), and A ( $n=3,1$ ST167, 1 ST617, 1 ST48). Serotyping revealed that out of the 21 ST131 E. coli, 19 had serotype O25:H4, and two had O16:H5 serogroup (Figure 1). All 19 ST131 E. coli with O25; H4 serogroup had fimH 30 alleles, whereas the two ST131 E. coli isolates with O16:H5 serogroup had
fimH41 allele, whereas the non-ST131 isolates exhibited diverse serotypes that included 19 different serotypes. Among them, the six emerging urosepsis pathogenic isolates belonging to ST405 had O102:H6 serogroup with fimH 29 allele except for one strain, which had fimH 27. The two isolates of high-risk clone ST410 had O8:H21 serogroup with fimH 24 allele. However, the three isolates belonging to the emerging pandemic lineage ST648 were not associated with any distinct sero or fim $H$ group. Similar to the results of serogroups, the non-ST131 isolates exhibited diverse fimH (10 different) types.

\section{Phenotypic and Genotypic Antimicrobial Resistance}

Majority of isolates were susceptible to amikacin (93\%), imipenem (93\%), and nitrofurantoin (83\%) (Figure 2). On the other hand, a majority of isolates were resistant to the common, empirically used antibiotics such as cefixime (96\%), cefuroxime (96\%), ceftriaxone (98\%), and ciprofloxacin (78\%). Besides, a moderate number of isolates were also resistant to cotrimoxazole (70\%), gentamicin (43\%), and cefepime (67\%). In contrast to the non-ST131 E. coli, isolates belonging to the ST131 lineage had higher resistance prevalence as the aggregate resistance score [median (range)] for ST131 isolates [6 (4-8)] was higher than that of non-ST131 isolates [5.5 (0-10)]. However, the difference was not statistically significant ( $p>0.5$ Mann-Whitney $U$-test).

Whole-genome sequencing (WGS) analysis identified 60 acquired AMR genes that are known to encode proteins conferring resistance to different classes of antibiotics including $\beta$-lactams, aminoglycosides, chloramphenicol, tetracycline, sulfonamides, trimethoprim, fluoroquinolone, and colistin (Figure 2).

\section{$\beta$-Lactam and Extended-Spectrum $\beta$-Lactamase Resistance}

We identified 24 genes associated with $\beta$-lactam resistance including $13 \mathrm{ESBL}, 10 \mathrm{AmpC} \beta$-lactamase, and 1 carbapenemase gene. The 13 ESBL genes included bla $a_{\mathrm{OXA}-1}(43 \%)$, bla $a_{\mathrm{TEM}-1 B}$ (28\%), bla $a_{\mathrm{CTX}-\mathrm{M}-27}(7 \%)$, and bla $a_{\mathrm{CTX}-\mathrm{M}-55}(4 \%)$. Thirty-eight of 46 isolates $(81 \%)$ carried the $b l a_{\mathrm{CTX}-\mathrm{M}-15}$ gene. Of 38 isolates carrying the bla $a_{\mathrm{CTX}}-\mathrm{M}-15$ gene, 36 were resistant to ceftriaxone and cefixime; therefore, this gene was strongly associated with third-generation cephalosporin resistance $(p<0.001)$. Moreover, the gene $b l a_{\mathrm{CTX}-\mathrm{M}-15}$ was detected in isolates of diverse genetic backgrounds affiliated to 6 different phylogroups and 13 different STs, including the high-risk clones-ST131 (18/21), ST405 (5/6), ST648 (3/3), ST410 (2/2), ST38 (1/1), ST73 (1/1), and ST1193 $(1 / 1)$. The $b l a_{\mathrm{CTX}}-\mathrm{M}-15$ gene was located on a plasmid for 23 of 38 isolates. Isolates also harbored other ESBL genes that included bla $a_{\mathrm{CTX}-\mathrm{M}-123}, b l a_{\mathrm{CTX}-\mathrm{M}-65}, b l a_{\mathrm{MIR}-3}, b l a_{\mathrm{OXA}-9}, b l a_{\mathrm{TEM}-169}$, $b l a_{\mathrm{TEM}-1 A}, b l a_{\mathrm{TEM}-1 B}, b l a_{\mathrm{TEM}-206}$, and bla $a_{\mathrm{TEM}-214}$ in lesser proportion. Similarly, a low prevalence of AmpC $\beta$-lactamase genes was detected (Figure 2).

\section{Aminoglycoside Resistance}

We identified 12 genes known to confer resistance to aminoglycosides (Figure 2). These included aac $\left(6^{\prime}\right)-\mathrm{Ib}-\mathrm{cr}$ (45\%), aadA5 (40\%), aac(3)-IIa (30\%), aph(3")-Ib (30\%), aph(6)-Id (30\%), and aadA2 (17\%). Of these six prominent 


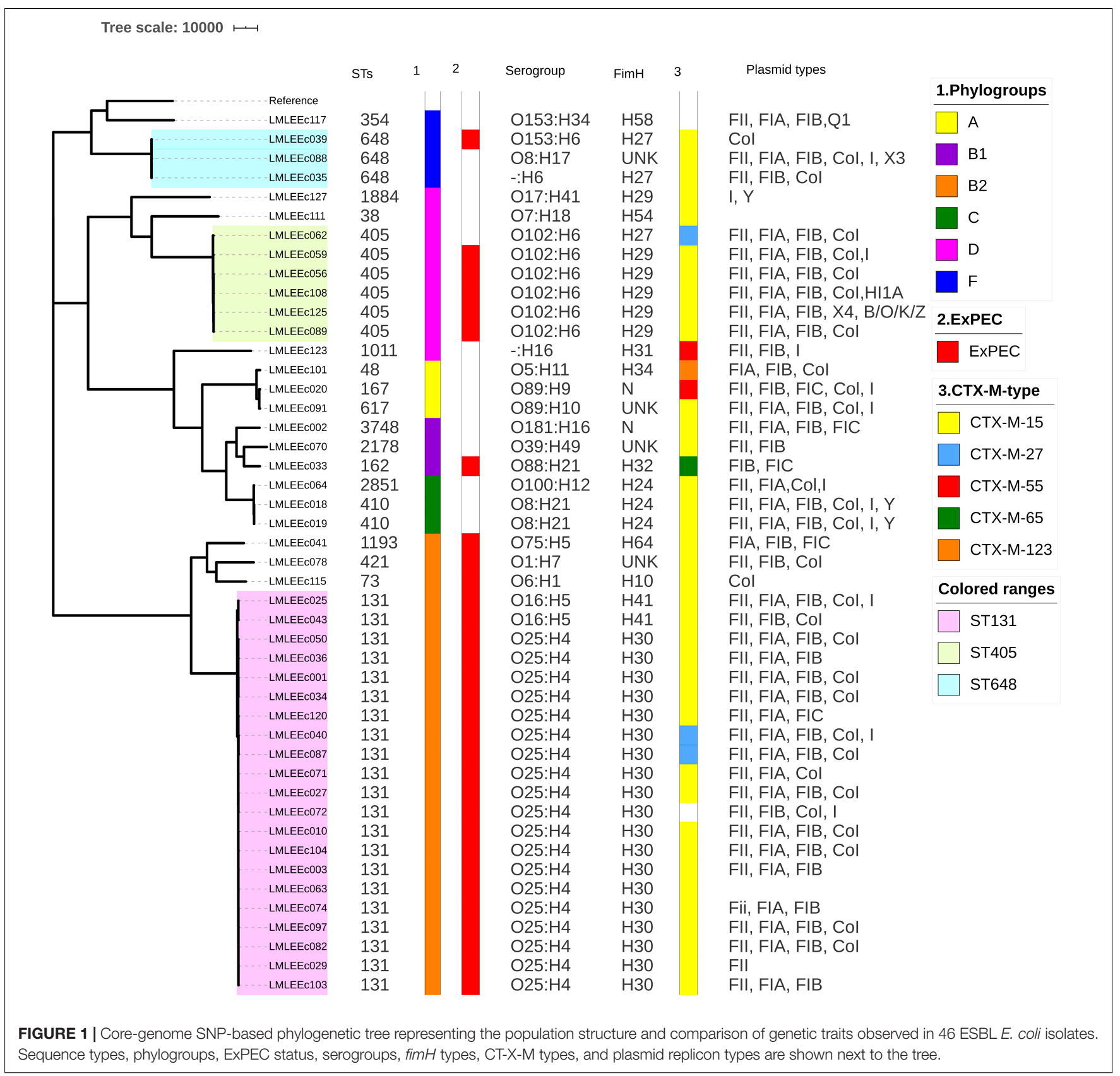

aminoglycoside genes, only two, $\operatorname{aac}(3)-I I a(14 / 13)$ and $\operatorname{aac}\left(6^{\prime}\right)$ Ib-cr (21/14), were strongly associated with gentamicin resistance $(p<0.001)$. None of the aminoglycoside genes was associated with amikacin resistance. Approximately $4 \%$ of the isolates harbored $\operatorname{aac}(3)-I I d$, aadA1, and $a p h\left(3^{\prime}\right)-I a$; other genes of this class included $a a c\left(6^{\prime}\right)-I b$ (one isolate), aadA2b (one isolate), and $\operatorname{armA}$ (one isolate).

\section{Cotrimoxazole Resistance}

Three genes known to encode sulfonamide resistance were identified: sul1 in 26/46 (57\%) isolates, sul2 in 14/46 (30\%) isolates, and sul3 in 2/46 (4\%) isolates. The presence of sul1 was positively correlated with phenotypic resistance to cotrimoxazole $(23 / 26)(p<0.001)$. Similarly, of the four genes responsible for trimethoprim resistance $[d f r A 17$ (43\%), dfrA12 (19\%), dfrA14 (4\%), dfrA5 (4\%)], the gene dfrA17 was strongly associated with cotrimoxazole resistance $(p<0.001)$.

\section{Fluoroquinolone Resistance}

Using PointFinder, we identified two mutations in gyr $A$ including S83L (serine to leucine) and D87N (aspartic acid to asparagine). The mutation S83L was detected in 13\% (6/46) of isolates. In contrast, $80 \%(37 / 46)$ of isolates had both the mutations (S83L and $\mathrm{D} 87 \mathrm{~N}$ ); the dual gyrA mutants were all resistant to ciprofloxacin. However, isolates (4/46) having a single mutation (S83L) were all susceptible to ciprofloxacin. Similarly, in the parC amino acid product, we identified two substitutions at codon position 80 (serine to isoleucine) and codon 84 (glutamic acid to 




FIGURE 2 | Heatmap showing the distribution of acquired AMR genes and AMR phenotypic profiles within 46 E. coli isolates. Gene names are listed on the left, and strain names are listed below the image. The presence of gene is indicated by colored blocks, and the gene absence is indicated by gray blocks. CRO, ceftriaxone; CXM, cefuroxime; CFM, cefixime; FEP, cefepime; NIT, nitrofurantoin; AMK, amikacin; CIP, ciprofloxacin; CoT, cotrimoxazole; IMP, imipenem; GEN, gentamicin.

valine). The mutation S80I was present in 33\% (15/46) of isolates, and the mutation E84V was detected in one isolate, whereas 22 of 46 isolates harbored both of these mutations, and they were all resistant to ciprofloxacin. Three substitutions were also detected in parE gene, with I529L being predominant (20/46) followed by S458A (11/46) and L416F (1/46). All isolates (20/46) carrying I529L mutation were ciprofloxacin-resistant, and this mutation was found in the majority of ST131 strains (18/21 ST131 strains). Additionally, the plasmid-mediated quinolone resistance gene $q n r s 1$ was detected in two isolates followed by qnrS4 and qnrS13 
in one isolate each. Except for qnrs13, isolates carrying qnrS genes were susceptible to ciprofloxacin.

\section{Carbapenem and Colistin Resistance}

NDM-5 carbapenemase was detected in four E. coli isolates $(8.5 \%)$. All four isolates belonged to the prominent clonal groups: two ST405 (phylogroup D), one ST648 (phylogroup F), and one ST167 (phylogroup A). All four $b l a_{N D M-5}$ genes were found to be located on plasmids (4/4 isolates). Except for one (ST167), all three isolates coharbored a $b l a_{\text {CTX }-\mathrm{M}-15}$ gene. Phenotypic resistance to imipenem was detected in only two of four bla $a_{\mathrm{NDM}-5}$-positive isolates. Nonetheless, all four bla $a_{\mathrm{NDM}-5}$-positive $E$. coli were pan-drug-resistant as resistance was detected to at least 7 of 10 antibiotics tested. We detected one $m c r-1$-positive E. coli belonging to ST1011; the $m c r-1$ gene was detected on a plasmid. This strain was coharboring a bla СТХM -55 gene and showed phenotypic resistance to cotrimoxazole, ciprofloxacin, ceftriaxone, cefuroxime, and cefixime and was notably susceptible to imipenem, gentamicin, and amikacin.

\section{Replicon Typing}

Screening of plasmid replicons among $46 E$. coli isolates using PlasmidFinder database detected a total of 12 plasmid replicons, which included FII, FIA, FIB, FIC, Col, I, X3, X4, Q1, B/O/K/Z, $\mathrm{HI}$, and Y (Figure 1). FII was the predominant replicon identified in $83 \%(38 / 46)$ of isolates followed by FIB, Col, and FIA replicon types, with $80 \%(37 / 46), 72 \%(33 / 46)$, and $70 \%(32 / 46)$, respectively. The mcr-1-positive E. coli harbored FII and FIB plasmid replicon types.

\section{Virulence Genes}

Figure 3 shows the distribution of virulence genes among the 46 sequenced E. coli isolates. A total of 32 of the 46 E. coli isolates were classified as ExPEC, which comprised 21/21 ST131 isolates (100\%) and 11/26 non-ST131 isolates (42\%). The ExPEC isolates identified were enriched with virulence genes such as pap, fim, sat, tia, hlyE, iutA, sitA, and fyuA. Isolates belonging to the ST131 clone showed higher aggregate virulence scores [median (range)], 22 (18-27) compared with non-ST131 isolates 14.5 (6-33). The only isolate that carried the highest virulence genes (33) belonged to ST73.

\section{Population Structure of Extended-Spectrum $\beta$-Lactamase Escherichia coli Isolates}

To investigate the relatedness of 46 ESBL E. coli isolates, single nucleotide polymorphism (SNP)-based core genome phylogenetic tree was constructed (Figure 1). The 46 E. coli genomes clustered with a good concordance to their phylogroups. Particularly, strains belonging to phylogroups B2, D, and F formed distinct clades, whereas strains belonging to phylogroups $\mathrm{A}, \mathrm{B} 1$, and $\mathrm{C}$ were clustered together under a large clade; 24 of these $46 \mathrm{E}$. coli isolates formed a large cluster of B2 phylogroup all qualified as ExPEC and had the most common plasmid combination of FII, FIA, and FIB; this clade mainly comprised (21/24) ST131 isolates. Further, it was identified that
16 of 21 ST131 E. coli belonged to the most virulent, widespread $\mathrm{H} 30 \mathrm{Rx}$ subclone, three belonged to $\mathrm{H} 30 \mathrm{R}$, and two belonged to fluoroquinolone-susceptible fim H 41, CTX-M-15-positive ST131 E. coli. The 21 ST131 E. coli isolates showed little or no difference in the SNP analysis; these isolates were collected over 15 months, which indicates widespread dissemination of this particular lineage. The sister clade of ST131 clustered E. coli lineages that included ST1193, ST73, and ST421. When the branches of nonST131 isolates were assessed closely, high clonality was observed between individual isolates in a few cases. For instance, six strains of ST405 formed an identical cluster; these were collected from November 2018 to July 2018, and five of six isolates carried bla $a_{\mathrm{CTX}-\mathrm{M}-15}$ and had identical serogroup and fimH types with almost common plasmid profile. However, two of these six isolates carried $b l a_{\mathrm{NDM}-5}$ gene in addition to the shared profiles. Similarly, ST410 isolates formed identical clusters with shared molecular profiles. However, ST648 strains formed an identical cluster but demonstrated diverse molecular profiles.

\section{DISCUSSION}

ESBL-producing E. coli is responsible for a significant number of nosocomial and community-acquired infections (Pana and Zaoutis, 2018). In this study, we used a WGS approach to analyze 46 ESBL-E. coli isolates collected over a year (16 months) from a referral diagnostic center in Dhaka, Bangladesh, a Southeast Asian country from which data such as these are acutely lacking.

Our study revealed that the ST131 lineage is by far the most prevalent lineage (45\%) represented particularly by H30Rx isolates (34\%). This dominance of ST131 E. coli in our study isolates is possibly because of our focus on ESBL-E. coli. However, the non-ST131 (55\%) E. coli isolates showed high diversity with respect to phylogroups, phylogenetic clustering, STs, serotypes, and fimH types, which is consistent with previous reports (Hussain et al., 2014; Musicha et al., 2017). Furthermore, we demonstrated that ESBLs, in particular, the CTX-M-type ESBLs, in Bangladesh have emerged across major high-risk international clones that comprise ST38, ST73, ST405, ST410, ST648, and ST1193 lineages, in addition to isolates of the pandemic E. coliST131.

Molecular typing revealed that a majority of CTX-M-type ESBL producers belonged to the ST131 lineage, which constituted the most clonal group in the population structure of the studied isolates. The predominance of this lineage and its association with CTX-M-15 is in line with the reports from worldwide studies (Nicolas-Chanoine et al., 2014), including those from Asia (Hussain et al., 2012; Ranjan et al., 2015). Further, we documented a greater proportion (76\%) of H30Rx subclones within the ST131 E. coli isolates, indicating a scenario of endemic circulation of H30Rx strains in the current setting, as these strains have been recovered between the span of April 2018 to June 2019. Clonal expansion of H30RX subclone might be the reason for the high prevalence and transmission of CTX-M-15-associated ST131 E. coli isolates in this setting. The H30Rx subclone is also reported in the neighboring country, India, in 2015 (Ranjan et al., 2015). The H30Rx isolates detected in this study were all qualified 


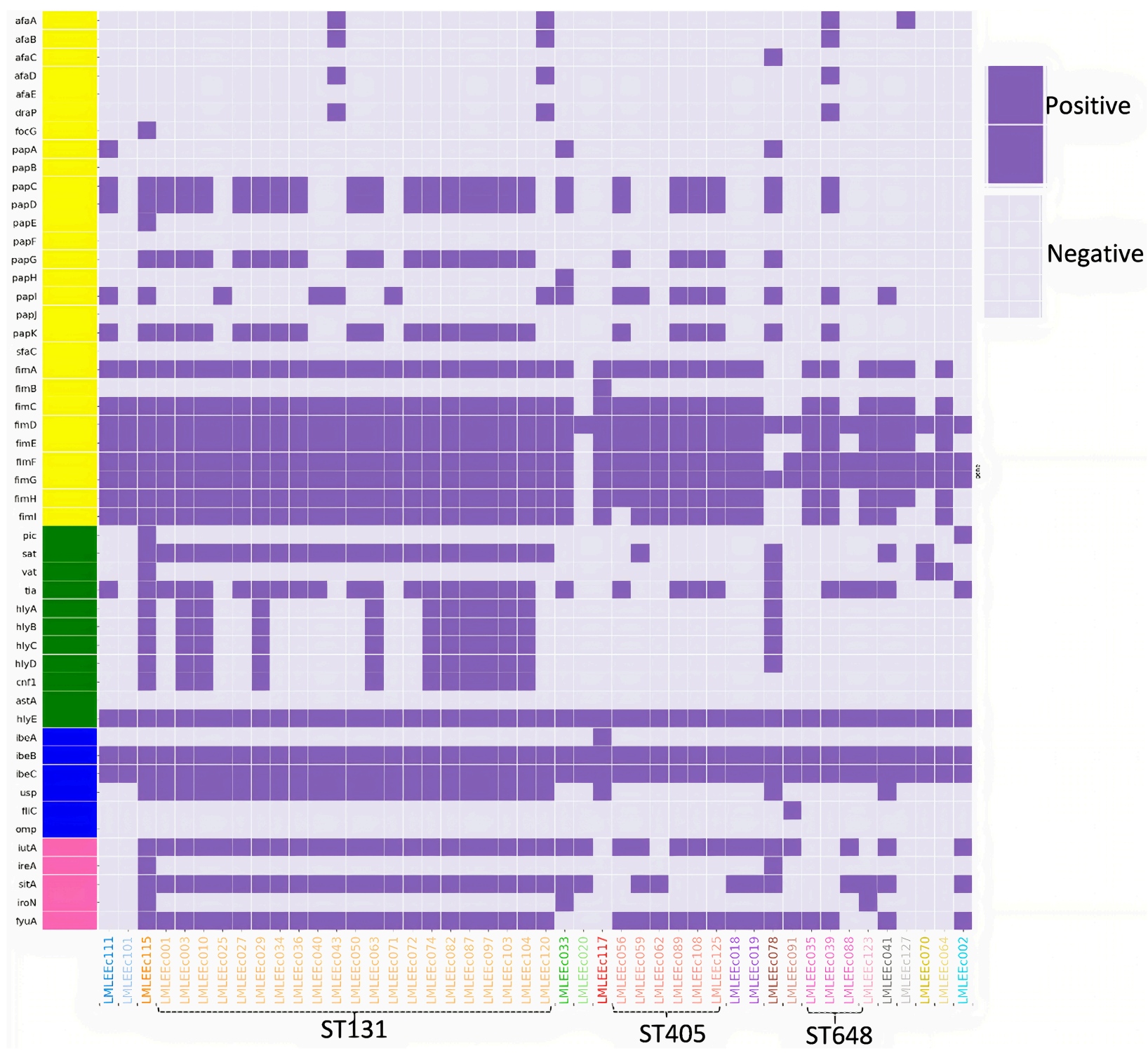

FIGURE 3 | Distribution of virulence gene profiles within 46 E. coli isolates are represented in the heatmap, with colored blocks indicating the gene presence and gray-colored blocks indicating the gene absence. Further, the virulence genes are vertically colored coded by functional categories (yellow, adhesins; blue, toxins; pink, miscellaneous; green, siderophores).

as ExPEC, which harbored a broad range of virulence genes with a relatively high virulence score, median (range): 27 (18-27). These isolates exhibited a high prevalence of a combination of IncFII, IncFIA, and IncFIB plasmid replicons, which is consistent with a recent WGS study from North Carolina (Kanamori et al., 2017). The gene $a a c\left(6^{\prime}\right)-l b$-cr encoding the aminoglycoside and fluoroquinolone-modifying enzyme was strongly associated with H30Rx strains. In addition to the bla $a_{\mathrm{CTX}-\mathrm{M}-15}$ gene, they exhibited a high prevalence of bla $\mathrm{OXA}_{-1}$ and high resistance to third-generation cephalosporin antibiotics (ceftriaxone and cefixime). The association of H30Rx subclone with extendedspectrum cephalosporin and fluoroquinolone resistance might have contributed to their predominance and epidemiological success over other $E$. coli lineages.
In this study, we have further shown that the $b a_{\mathrm{CTX}}-\mathrm{M}-15$ gene exists in genetically diverse strains including the prominent non-ST131 uropathogenic E. coli lineages such as ST38, ST73, ST405, ST410, ST648, and ST1193. Isolates of ST38 were reported to be evolving and is described to be increasingly detected in UTIs, which was previously considered to be a gut pathogen because they harbor genes for both EAEC and ExPEC (Chattaway et al., 2014). It is noticed that the ST38 isolate identified in this study was moderately lower in virulence and AMR gene content; particularly, it lacked the fluoroquinolone resistance and plasmids. Nonetheless, they were identified as ExPEC. ST73 is another frequently isolated ExPEC from UTI and bloodstream infections (Riley, 2014). The ST73 isolate in our study was found to carry an extensive array of virulence genes, whereas it was 
susceptible to most of the antibiotics and carried only IncCoI plasmid type, in contrast to other studies, which detected FII, FIA, and FIB plasmids; the reason for this could be that the ST73 strains are not expanding clonally as also suggested by others (Bogema et al., 2020).

ST405 E. coli lineage has been implicated as drivers of $b l a_{\mathrm{CTXM}-15}$ and is often associated with $b l a_{\mathrm{NDM}}$ genes and extensive virulence repertoire similar to the ST131 (Devanga Ragupathi et al., 2020). All six ST405 isolates from our study demonstrated high AMR rates and specifically carried a set of common plasmid replicons, including IncFII, IncFIA, IncFIB, and IncCoI. Five of these six ST405 strains qualified as ExPEC with extensive virulence gene profiles, consistent with other reports (Zhang et al., 2018; Devanga Ragupathi et al., 2020). Two of these six ST405 isolates also harbored $b l a_{\mathrm{NDM}-5}$ gene. ST410 is considered another emerging "highrisk" clone that is resistant to fluoroquinolones, cephalosporins, and sometimes carbapenems (Roer et al., 2018). The two ST410 isolates in our collection showed moderate virulence and resistance profiles; they harbored $b l_{\mathrm{CTX}-\mathrm{M}-15}$ gene with a set of five plasmid replicon types and fimH 24 allele (Figure 1). The lineage ST648 has been predicted to become another internationally circulating clone that will worsen infection treatment possibilities because of its AMR (Schaufler et al., 2019). The three ST648 isolates detected in this study carried bla $a_{\mathrm{CTX}-\mathrm{M}-15}$ and demonstrated high AMR rates; all the three strains consistently carried IncCoI plasmids. One ST648 strain was also positive for the $b l a_{\mathrm{NDM}-5}$ gene. ST1193 is the latest pandemic multidrug-resistant uropathogenic clonal group (Johnson et al., 2019), which is usually detected among nonlactose fermenters (Johnson et al., 2019). The ST1193 isolate in our collection was qualified as ExPEC; it showed high resistance rates, with particular resistance to fluoroquinolones, cephalosporins, and cotrimoxazole. It harbored IncFIA, IncFIB, and IncCoI plasmid types.

Several WGS studies have described the features of E. coli ST131. In contrast, not many have addressed the molecular epidemiology of non-ST131 E. coli worldwide. In this study, we have described that the ST131 E. coli is currently the most prevalent CTX-M-type ESBL producer in our community. However, we have also drawn attention to the emergence of significant clonal groups among non-ST131 E. coli. Further work is warranted in Bangladesh to accurately estimate the prevalence of these clonal groups and systematically analyze them

\section{REFERENCES}

Bankevich, A., Nurk, S., Antipov, D., Gurevich, A. A., Dvorkin, M., Kulikov, A. S., et al. (2012). SPAdes: a new genome assembly algorithm and its applications to single-cell sequencing. J. Comput. Biol. 19, 455-477. doi: 10.1089/cmb.2012. 0021

Baquero, F., Tedim, A. P., and Coque, T. M. (2013). Antibiotic resistance shaping multi-level population biology of bacteria. Front. Microbiol. 4:15. doi: 10.3389/ fmicb.2013.00015

Beghain, J., Bridier-Nahmias, A., Le Nagard, H., Denamur, E., and Clermont, O. (2018). ClermonTyping: an easy-to-use and accurate in silico method for Escherichia genus strain phylotyping. Microb. Genomics 4:192. doi: 10.1099/ mgen.0.000192 in a global context, which is pertinent from public health and clinical standpoint.

Taken together, our study suggests that the CTX-M-type ESBLs and particularly the CTX-M-15 are prevalent among diverse E. coli STs circulating in Dhaka, Bangladesh. Further, our findings confirm the striking predominance of ST131 lineage and also revealed the presence of several major high-risk nonST131 ExPEC clonal lineages, such as ST38, ST73, ST405, ST410, ST648, and ST1193, all associated with cephalosporin resistance and virulence. Further genomic epidemiological studies are needed to keep track of significant virulent/multiresistant E. coli clones in Bangladesh. Such studies are needed to inform us about the evolutionary dynamics of pathogenicity and resistance in emerging $E$. coli lineages of public health concern which will aid in evidence-based infection control and antibioticprescribing policies.

\section{DATA AVAILABILITY STATEMENT}

The datasets generated for this study can be found in the GenBank (Bioproject Accession: PRJNA654992). The GenBank accession numbers of 46 genomes sequenced in this study can be found in Table 1.

\section{AUTHOR CONTRIBUTIONS}

$\mathrm{AH}$ and RM designed the study, performed genome sequencing, and drafted the manuscript. AA, AH, and RM carried out the bioinformatics analyses and interpretation of results. MI, MS, $\mathrm{SM}, \mathrm{AT}, \mathrm{FH}$, and NA were involved in the culture and AST of bacteria isolates. DA and DM supervised the study. All authors have read and approved the final manuscript.

\section{ACKNOWLEDGMENTS}

This research study was funded by core donors who provide unrestricted support to icddr,b for its operations and research. Current donors providing unrestricted support include the governments of Bangladesh, Canada, Sweden, and the United Kingdom. We gratefully acknowledge these donors for their support and commitment to icddr,b's research efforts.

Begum, N., and Shamsuzzaman, S. M. (2016). Emergence of CTX-M-15 producing E. coli O25b-ST131 clone in a tertiary care hospital of Bangladesh. Malays. J. Pathol. 38, 241-249.

Bogema, D. R., McKinnon, J., Liu, M., Hitchick, N., Miller, N., Venturini, C., et al. (2020). Whole-genome analysis of extraintestinal Escherichia coli sequence type 73 from a single hospital over a 2 year period identified different circulating clonal groups. Microb. Genom. 6:e000255. doi: 10.1099/mgen.0.0 00255

Bolger, A. M., Lohse, M., and Usadel, B. (2014). Trimmomatic: a flexible trimmer for Illumina sequence data. Bioinformatics 30, 2114-2120. doi: 10. 1093/bioinformatics/btu170

Calbo, E., Romaní, V., Xercavins, M., Gómez, L., Vidal, C. G., Quintana, S., et al. (2006). Risk factors for community-onset urinary tract infections due 
to Escherichia coli harbouring extended-spectrum $\beta$-lactamases. J. Antimicrob. Chemother. 57, 780-783. doi: 10.1093/jac/dkl035

Cantón, R., and Coque, T. M. (2006). The CTX-M beta-lactamase pandemic. Curr. Opin. Microbiol. 9, 466-475.

Carattoli, A., Zankari, E., García-Fernández, A., Voldby Larsen, M., Lund, O., Villa, L., et al. (2014). In silico detection and typing of plasmids using plasmidfinder and plasmid multilocus sequence typing. Antimicrob. Agents Chemother. 58, 3895-3903. doi: 10.1128/AAC.02412- 14

Chen, L., Yang, J., Yu, J., Yao, Z., Sun, L., Shen, Y., et al. (2005). VFDB: a reference database for bacterial virulence factors. Nucleic Acids Res. 33, D325-D328. doi: 10.1093/nar/gki008

Chen, S. L., Ding, Y., Apisarnthanarak, A., Kalimuddin, S., Archuleta, S., Omar, S. F. S., et al. (2019). The higher prevalence of extended spectrum betalactamases among Escherichia coli ST131 in Southeast Asia is driven by expansion of a single, locally prevalent subclone. Sci. Rep. 9:13245. doi: 10.1038/ s41598-019-49467-5

Clinical and Laboratory Standards Institute (2015). Performance Standards for Antimicrobial Susceptibility Testing; 20th Informational Supplement M100-S25. Wayne, PA: Clinical and Laboratory Standards Institute.

Croucher, N. J., Page, A. J., Connor, T. R., Delaney, A. J., Keane, J. A., Bentley, S. D., et al. (2015). Rapid phylogenetic analysis of large samples of recombinant bacterial whole genome sequences using Gubbins. Nucleic Acids Res. 43:e15. doi: 10.1093/nar/gku1196

Doi, Y. (2019). Treatment options for carbapenem-resistant gram-negative bacterial infections. Clin. Infect. Dis. 69, S565-S575. doi: 10.1093/cid/c iz830

Franz, E., Veenman, C., van Hoek, A. H. A. M., Husman, A., de, R., Blaak, H., et al. (2015). Pathogenic Escherichia coli producing extended-spectrum $\beta$-lactamases isolated from surface water and wastewater. Sci. Rep. 5:14372. doi: 10.1038/ srep14372

Hussain, A., Ewers, C., Nandanwar, N., Guenther, S., Jadhav, S., Wieler, L. H., et al. (2012). Multiresistant uropathogenic Escherichia coli from a region in India where urinary tract infections are endemic: genotypic and phenotypic characteristics of sequence type 131 isolates of the CTX-M-15 extendedspectrum- $\beta$-lactamase-producing lineage. Antimicrob. Agents Chemother. 56, 6358-6365. doi: 10.1128/AAC.01099- 12

Hussain, A., Ranjan, A., Nandanwar, N., Babbar, A., Jadhav, S., and Ahmed, N. (2014). Genotypic and phenotypic profiles of Escherichia coli isolates belonging to clinical sequence type 131 (ST131), clinical non-ST131, and fecal non-ST131 lineages from India. Antimicrob. Agents Chemother. 58, 7240-7249. doi: 10. 1128/AAC.03320-14

Joensen, K. G., Tetzschner, A. M. M., Iguchi, A., Aarestrup, F. M., and Scheutz, F. (2015). Rapid and easy in silico serotyping of Escherichia coli isolates by use of whole-genome sequencing data. J. Clin. Microbiol. 53, 2410-2426. doi: 10.1128/JCM.00008-15

Johnson, J. R., and Stell, A. L. (2000). Extended virulence genotypes of Escherichia coli strains from patients with urosepsis in relation to phylogeny and host compromise. J. Infect. Dis. 181, 261-272. doi: 10.1086/315217

Johnson, T. J., Elnekave, E., Miller, E. A., Munoz-Aguayo, J., Figueroa, C. F., Johnston, B., et al. (2019). Phylogenomic analysis of extraintestinal pathogenic Escherichia coli sequence type 1193, an emerging multidrug-resistant clonal group. Antimicrob. Agents Chemother. 63:e1913-18. doi: 10.1128/AAC.0191318

Kanamori, H., Parobek, C. M., Juliano, J. J., Johnson, J. R., Johnston, B. D., Johnson, T. J., et al. (2017). Genomic analysis of multidrug-resistant Escherichia coli from north carolina community hospitals: ongoing circulation of CTXM-producing ST131-H30Rx and ST131-H30R1 strains. Antimicrob. Agents Chemother. 61:e00912-17. doi: 10.1128/AAC.00912-17

Kumarasamy, K. K., Toleman, M. A., Walsh, T. R., Bagaria, J., Butt, F., Balakrishnan, R., et al. (2010). Emergence of a new antibiotic resistance mechanism in India, Pakistan, and the UK: a molecular, biological, and epidemiological study. Lancet Infect. Dis. 10, 597-602. doi: 10.1016/S14733099(10)70143-2

Larsen, M. V., Cosentino, S., Rasmussen, S., Friis, C., Hasman, H., Marvig, R. L., et al. (2012). Multilocus sequence typing of total-genome-sequenced bacteria. J. Clin. Microbiol. 50, 1355-1361. doi: 10.1128/JCM.06094-11

Lina, T. T., Khajanchi, B. K., Azmi, I. J., Islam, M. A., Mahmood, B., Akter, M., et al. (2014). Phenotypic and molecular characterization of extended-spectrum beta-lactamase-producing Escherichia coli in Bangladesh. PLoS One 9:e108735. doi: 10.1371/journal.pone.0108735

Chattaway, M. A., Jenkins, C., Ciesielczuk, H., Day, M., DoNascimento, V., Day, M., et al. (2014). Evidence of evolving extraintestinal enteroaggregative Escherichia Coli ST38 clone. Emerg. Infect. Dis. 20, 1935-1937. doi: 10.3201/ EID2011.131845

Manges, A. R., Geum, H. M., Guo, A., Edens, T. J., Fibke, C. D., and Pitout, J. D. D. (2019). Global extraintestinal pathogenic escherichia coli (Expec) lineages. Clin. Microbiol. Rev. 32:e0135-18. doi: 10.1128/CMR.00135-18

Mathers, A. J., Peirano, G., and Pitout, J. D. D. (2015). The role of epidemic resistance plasmids and international high-risk clones in the spread of multidrug-resistant Enterobacteriaceae. Clin. Microbiol. Rev. 28, 565-591. doi: 10.1128/CMR.00116-14

Mazumder, R., Abdullah, A., Ahmed, D., and Hussain, A. (2020a). High prevalence of blactx-m-15 gene among extended-spectrum $\beta$-lactamaseproducing escherichia coli isolates causing extraintestinal infections in bangladesh. Antibiotics 9:796. doi: 10.3390/antibiotics9110796

Mazumder, R., Abdullah, A., Hussain, A., Ahmed, D., and Mondal, D. (2020b). Draft genome sequence of chromobacterium violaceum RDN09, isolated from a patient with a wound infection in bangladesh. Microbiol. Resour. Announc. 9:e00957-20. doi: 10.1128/mra.00957-20

McArthur, A. G., Waglechner, N., Nizam, F., Yan, A., Azad, M. A., Baylay, A. J., et al. (2013). The comprehensive antibiotic resistance database. Antimicrob. Agents Chemother 57, 3348-3357. doi: 10.1128/AAC.00419-13

Mikheenko, A., Prjibelski, A., Saveliev, V., Antipov, D., and Gurevich, A. (2018). Versatile genome assembly evaluation with QUAST-LG. Bioinformatics 34, i142-i150. doi: 10.1093/bioinformatics/bty266

Musicha, P., Feasey, N. A., Cain, A. K., Kallonen, T., Chaguza, C., Peno, C., et al. (2017). Genomic landscape of extended-spectrum $\beta$-lactamase resistance in Escherichia coli from an urban African setting. J. Antimicrob. Chemother. 72, 1602-1609. doi: 10.1093/jac/dkx058

Nicolas-Chanoine, M.-H., Bertrand, X., and Madec, J.-Y. (2014). Escherichia coli ST131, an intriguing clonal group. Clin. Microbiol. Rev. 27, 543-574. doi: 10. 1128/CMR.00125-13

Nicolas-Chanoine, M. H., Blanco, J., Leflon-Guibout, V., Demarty, R., Alonso, M. P., Caniça, M. M., et al. (2008). Intercontinental emergence of Escherichia coli clone O25:H4-ST131 producing CTX-M-15. J. Antimicrob. Chemother. 61, 273-281. doi: 10.1093/jac/dkm464

Ragupathi, N. K. D., Veeraraghavan, B., Sethuvel, D. P. M., Anandan, S., Vasudevan, K., Neerav, A. R., et al. (2020). First Indian report on genome-wide comparison of multidrug-resistant Escherichia Coli from blood stream infections. PLoS One 15:e220428. doi: 10.1371/JOURNAL.PONE.02 20428

Pana, Z. D., and Zaoutis, T. (2018). Treatment of extended-spectrum $\beta$-lactamaseproducing Enterobacteriaceae (ESBLs) infections: what have we learned until now? F1000 Res. 7:F1000 Faculty Rev-1347. doi: 10.12688/F1000RESEARCH. 14822.1

Paterson, D. L., and Bonomo, R. A. (2005). Extended-spectrum $\beta$-lactamases: a clinical update. Clin. Microbiol. Rev. 18, 657-686. doi: 10.1128/CMR.18.4.657686.2005

Pitout, J. D., and Laupland, K. B. (2008). Extended-spectrum $\beta$-lactamaseproducing Enterobacteriaceae: an emerging public-health concern. Lancet Infect. Dis. 8, 159-166. doi: 10.1016/S1473-3099(08)70041-0

Price, L. B., Johnson, J. R., and Aziz, M. (2013). The epidemic of extendedspectrum- $\beta$-lactamase-producing Escherichia coli ST131 is driven by a single highly pathogenic subclone. H 30-Rx. MBio 4:e00377-13. doi: 10.1128/mBio. 00377-13.Editor

Ranjan, A., Shaik, S., Hussain, A., Nandanwar, N., Semmler, T., Jadhav, S., et al. (2015). Genomic and functional portrait of a highly virulent, CTX-M-15producing H 30-Rx subclone of Escherichia coli sequence type 131. Antimicrob. Agents Chemother. 59, 6087-6095. doi: 10.1128/AAC.01447-15

Riley, L. W. (2014). Pandemic lineages of extraintestinal pathogenic Escherichia coli. Clin. Microbiol. Infect. 20, 380-390. doi: 10.1111/1469-0691.12646

Roer, L., Overballe-Petersen, S., Hansen, F., Schønning, K., Wang, M., Røder, B. L., et al. (2018). Escherichia coli sequence type 410 is causing new international high-risk clones. mSphere 3:e00337-18. doi: 10.1128/MSPHERE.00337-18

Roer, L., Tchesnokova, V., Allesoe, R., Muradova, M., Chattopadhyay, S., Ahrenfeldt, J., et al. (2017). Development of a web tool for Escherichia coli 
subtyping based on fimh alleles. J. Clin. Microbiol. 55, 2538-2543. doi: 10.1128/ JCM.00737-17

Schaufler, K., Semmler, T., Wieler, L. H., Trott, D. J., Pitout, J., Peirano, G., et al. (2019). Genomic and functional analysis of emerging virulent and multidrug-resistant Escherichia coli lineage sequence type 648. Antimicrob. Agents Chemother. 63:e00243-19. doi: 10.1128/AAC.00243-19

Seemann, T. (2014). Prokka: rapid prokaryotic genome annotation. Bioinformatics 30, 2068-2069. doi: 10.1093/bioinformatics/btu153

Seemann, T. (2015). Snippy: Rapid Haploid Variant Calling and Core SNP Phylogeny. San Francisco, CA: GitHub.

Shaik, S., Ranjan, A., Tiwari, S. K., Hussain, A., Nandanwar, N., Kumar, N., et al. (2017). Comparative genomic analysis of globally dominant ST131 clone with other epidemiologically successful extraintestinal pathogenic Escherichia coli (ExPEC) lineages. MBio 8:e01596-17. doi: 10.1128/mBio.01596-17

Stamatakis, A. (2014). RAxML Version 8: A Tool for Phylogenetic Analysis and Post-Analysis of Large Phylogenies.

Stoesser, N., Sheppard, A. E., Moore, C. E., Golubchik, T., Parry, C. M., Nget, P., et al. (2015). Extensive within-host diversity in fecally carried extendedspectrum-beta-lactamase-producing Escherichia coli Isolates: Implications for transmission analyses. J. Clin. Microbiol. 53, 2122-2131. doi: 10.1128/JCM. 00378- 15

Zankari, E., Allesøe, R., Joensen, K. G., Cavaco, L. M., Lund, O., and Aarestrup, F. M. (2017). PointFinder: a novel web tool for WGS-based detection of antimicrobial resistance associated with chromosomal point mutations in bacterial pathogens. J. Antimicrob. Chemother. 72, 2764-2768. doi: 10.1093/jac/ dkx217

Zhang, X., Feng, Y., Zhou, W., McNally, A., and Zong, Z. (2018). Cryptic transmission of ST405 Escherichia coli carrying bla NDM-4 in hospital. Sci. Rep. 8:390. doi: 10.1038/s41598-017-18910-w

Conflict of Interest: The authors declare that the research was conducted in the absence of any commercial or financial relationships that could be construed as a potential conflict of interest.

Publisher's Note: All claims expressed in this article are solely those of the authors and do not necessarily represent those of their affiliated organizations, or those of the publisher, the editors and the reviewers. Any product that may be evaluated in this article, or claim that may be made by its manufacturer, is not guaranteed or endorsed by the publisher.

Copyright (c) 2021 Mazumder, Hussain, Abdullah, Islam, Sadique, Muniruzzaman, Tabassum, Halim, Akter, Ahmed and Mondal. This is an open-access article distributed under the terms of the Creative Commons Attribution License (CC BY). The use, distribution or reproduction in other forums is permitted, provided the original author(s) and the copyright owner(s) are credited and that the original publication in this journal is cited, in accordance with accepted academic practice. No use, distribution or reproduction is permitted which does not comply with these terms. 